\title{
Autonomous Effects of Shear Stress and Cyclic Circumferential Stretch regarding Endothelial Dysfunction and Oxidative Stress: An ex vivo Arterial Model
}

\author{
Tyler N. Thacher ${ }^{\mathrm{a}}$ Paolo Silacci $^{\mathrm{d}}$ Nikos Stergiopulos ${ }^{\mathrm{a}}$ Rafaela F. da Silva ${ }^{\mathrm{a}-\mathrm{c}}$ \\ a Laboratory of Hemodynamics and Cardiovascular Technology, Swiss Federal Institute of Technology, Lausanne, \\ ${ }^{b}$ Department of Neurosurgery, Geneva University Hospital, and ${ }^{\mathrm{C}}$ Department of Neurosciences, University Medical \\ Center, University of Geneva Medical School, Geneva, and ${ }^{\mathrm{d}}$ Station de recherche Agroscope Liebefeld, \\ Posieux, Switzerland
}

\section{Key Words}

Endothelial dysfunction $\cdot$ Nitrotyrosine $\cdot$ Oxidative stress •

Cyclic stretch and shear stress

\begin{abstract}
Cyclic circumferential stretch and shear stress caused by pulsatile blood flow work in concert, yet are very different stimuli capable of independently mediating endothelial function by modulating eNOS expression, oxidative stress (via production of superoxide anion) and NO bioavailability. Porcine carotid arteries were perfused using an ex vivo arterial support system for $72 \mathrm{~h}$. Groups we created by combining normal (5\%) and reduced (1\%) stretch with high shear (6 \pm 3 dynes $\left./ \mathrm{cm}^{2}\right)$ and oscillatory shear $\left(0.3 \pm 3\right.$ dynes $\left./ \mathrm{cm}^{2}\right)$ stress while maintaining a pulse pressure of $80 \pm 10 \mathrm{~mm} \mathrm{Hg}$. Oscillatory flow and reduced stretch both proved detrimental to endothelial function, whereas oscillatory flow alone dominated total endogenous vascular wall superoxide anion production. Yet, when superoxide anion production was analyzed in just the endothelial region, we observed that it was
\end{abstract}

modulated more significantly by reduced cyclic stretch than by oscillatory shear, emphasizing an important distinction between shear-and stretch-mediated effects to the vascular wall. Western blotting analysis of eNOS and nitrotyrosine proved that they too are more significantly negatively modulated by oscillatory flow than by reduced stretch. These findings point out how shear and stretch stimulate regions of the vascular wall differently, affecting NO bioavailability and contributing to vascular disease.

Copyright $\odot 2009$ S. Karger AG, Basel

\section{Introduction}

Within the vasculature, endothelial cells are constantly exposed to dynamic mechanical forces generated by pulsatile blood flow. Two such stimuli known to modulate endothelial function are shear stress and cyclic circumferential strain. It has been documented that areas of the vasculature exposed to low mean shear stress and cyclic reversal of flow direction (also called oscillatory flow,

\section{KARGER}

Fax +41613061234 E-Mail karger@karger.ch www.karger.com
(C) 2009 S. Karger AG, Basel

$1018-1172 / 10 / 0474-0336 \$ 26.00 / 0$

Accessible online at:

www.karger.com/jvr
Dr. Tyler Thacher

Laboratoire d'Hémodynamique et de Technologie Cardiovasculaire

École Polytechnique Fédéral de Lausanne

Bâtiment AI 1241, Station 15, CH-1015 Lausanne (Switzerland)

Tel. +41 21693 9654, Fax +41 21693 9635, E-Mail tyler.thacher@ epfl.ch 
OSC) are at higher risk of developing endothelial dysfunction than those exposed to a unidirectional high shear stress (HSS) [1-4]. Similarly, it has also been suggested that reduction of arterial compliance may contribute to endothelial dysfunction, since it has been linked to diseases such as atherosclerosis and hypertension $[5,6]$. Yet, in most studies these 2 stimuli are simultaneously coupled in vivo, making it very difficult to understand their individual contributions. Some attempts have been made to decouple stretch and shear stress in vitro by using different cell lines in a variety of stretch systems and flow chambers, straying from reality and making it hard to draw definitive conclusions. In this study we wish to find a compromise between the in vivo and in vitro work of the past by studying the independent effects of shear stress and cyclic stretch and how they contribute to endothelial dysfunction.

The underlying mechanisms of endothelial dysfunction are numerous and remain unclear, yet it is generally agreed upon that the most dangerous revolve around a central point, that is, diminished bioavailability of nitric oxide (NO) [7]. One mechanism involves the downregulation of endothelial NO synthase (eNOS) [8] expression, an enzyme produced by the endothelium which upon conversion of L-arginine to L-citrulline produces NO. Another mechanism thought to significantly affect NO bioavailability involves the upregulation of vascular levels of reactive oxygen species (ROS). Elevated levels of ROS are known to damage vascular tissue as well as react with $\mathrm{NO}$, forming peroxynitrite [9], thus removing NO [10] from the vasculature and contributing to pro-atherogenic conditions [11]. The predominant system producing ROS in vascular smooth muscle and endothelial cells is the membrane-bound $\mathrm{NAD}(\mathrm{P}) \mathrm{H}$ oxidase (NOX), regulated by the expression of p22-phox [12] and p47-phox [13].

The goal of this study is to understand how shear stress and cyclic stretch individually mediate the expression of eNOS, the interaction of ROS and NO and if these factors contribute to endothelial dysfunction in porcine arterial tissue perfused ex vivo.

\section{Methods}

\section{Arterial Groups}

Left internal carotid arteries of 6-month-old pigs weighing $120-150 \mathrm{~kg}$ were obtained from the local slaughterhouse (Bell SA). Adventitial tissue was removed and a $3.5-\mathrm{cm}$ segment, $1 \mathrm{~cm}$ distal to the bifurcation, was excised. The arterial segments were then mounted onto the ex vivo arterial support system (see de- scription below). The segments were stretched longitudinally to 1.3 times the unstretched and unpressurized length. To simulate decreased compliance, a silicon cuff (Statice Sante) of 6.0 or 8.0 $\mathrm{mm}$ (depending on the outer diameter) and of $0.2 \pm 0.05 \mathrm{~mm}$ thickness was placed around the arterial segment. The reduction in circumferential cyclic stretch obtained with the cuff was roughly $80 \%$, when compared to the uncuffed arterial segment.

\section{Ex vivo Arterial Perfusion System}

The ex vivo arterial perfusion system used in this study enables the perfusion of isolated arterial segments under precise control of perfusion pressure and flow. Details on ex vivo arterial support system have been given previously [14]. The arterial segments were perfused for $72 \mathrm{~h}$ with a medium cocktail, as described previously [15]. Perfusion flow was adapted to create either a pulsatile unidirectional HSS with a mean value of 6 dyne $/ \mathrm{cm}^{2}$ and amplitude of 3 dyne $/ \mathrm{cm}^{2}$, or an oscillatory shear stress with a mean value of $0.3 \mathrm{dyne} / \mathrm{cm}^{2}$ and amplitude of $3 \mathrm{dyne} / \mathrm{cm}^{2}$, both with a frequency of $1 \mathrm{~s}$. Perfusion pressure was set to $80 \mathrm{~mm} \mathrm{Hg}$ with pulse pressure amplitude of $\pm 10 \mathrm{~mm} \mathrm{Hg}$. Resulting strains were $4-5 \%$ for the uncuffed segment, which is in the physiological range of pulsatile stretch for the porcine carotid, and less than $1 \%$ for the cuffed segment, simulating a less compliant arterial segment.

\section{Endothelial Cell Functionality Analysis}

Arterial rings were tested before and after the perfusion experiment to determine the capacity to achieve endothelial-mediated vasorelaxation. Arterial rings were mounted in an organ chamber (EMKA Technology), equilibrated in a Krebs solution at $37^{\circ} \mathrm{C}$, infused with $5 \% \mathrm{CO}_{2}$ and $95 \% \mathrm{O}_{2}$. Resting tension was adjusted to $2 \mathrm{~g}$. Arterial rings were precontracted with $80 \mathrm{mM}$ $\mathrm{KCl}$ until a constant maximum contraction was reached. Then a $10^{-6}-\mathrm{M}$ dose of norepinephrine was introduced, achieving a contraction $50 \%$ as large as that achieved with KCL. Next, doses of bradykinin (BK) from $10^{-11}$ to $10^{-8} \mathrm{M}$ were given and dose-response curves for BK-mediated relaxation were calculated. Doseresponse curves for sodium nitroprusside were also obtained.

\section{Immunofluorescence and Dihydroethidium}

After the perfusion, a 5-mm segment of the artery was rinsed with $0.9 \% \mathrm{NaCl}$, snap-frozen in OCT compound (Tissue-Tek) and stored at $-80^{\circ} \mathrm{C}$ for further analysis. For p22-phox and nitrotyrosine staining, sections of $5 \mu \mathrm{m}$ were cut, air-dried and fixed in $100 \%$ acetone for $5 \mathrm{~min}$ at $-20^{\circ} \mathrm{C}$. Sections were permeabilized with $0.1 \%$ Triton X-100 in PBS for $10 \mathrm{~min}$, then incubated for 60 min with rabbit anti-p22 ${ }^{\text {phox }}$ (p22-phox, 1:500; Santa Cruz Biotechnology) or mouse anti-nitrotyrosine (nitrotyrosine, 1:100; Abcam) in 10\% normal goat serum in PBS. The p22-phox sections were then incubated with Alexa Fluor 568 goat anti-rabbit IgG (Invitrogen) as a secondary antibody for $45 \mathrm{~min}$. Sections were examined on a Zeiss Axiovert 135 microscope at $20 \times$ magnification. For nitrotyrosine, sections were incubated with ECL-peroxidase-labeled anti-mouse (1:1,000; Amersham) as a secondary antibody and counterstained using a DAB substrate kit (Vector Laboratories). Sections were examined on an Olympus AX 70 at $10 \times$ magnification. All steps were performed at room temperature.

For dihydroethidium (DHE) detection, 5- $\mu \mathrm{m}$ arterial cryosections (as described above) were incubated for $30 \mathrm{~min}$ at $37^{\circ} \mathrm{C}$ 
Fig. 1. BK dose-dependent relaxation capacity of porcine carotid arterial segments after $72 \mathrm{~h}$ of ex vivo perfusion. Data are represented as means $\pm \mathrm{SD}, \mathrm{n}=6,{ }^{*} \mathrm{p}<$ 0.05 .

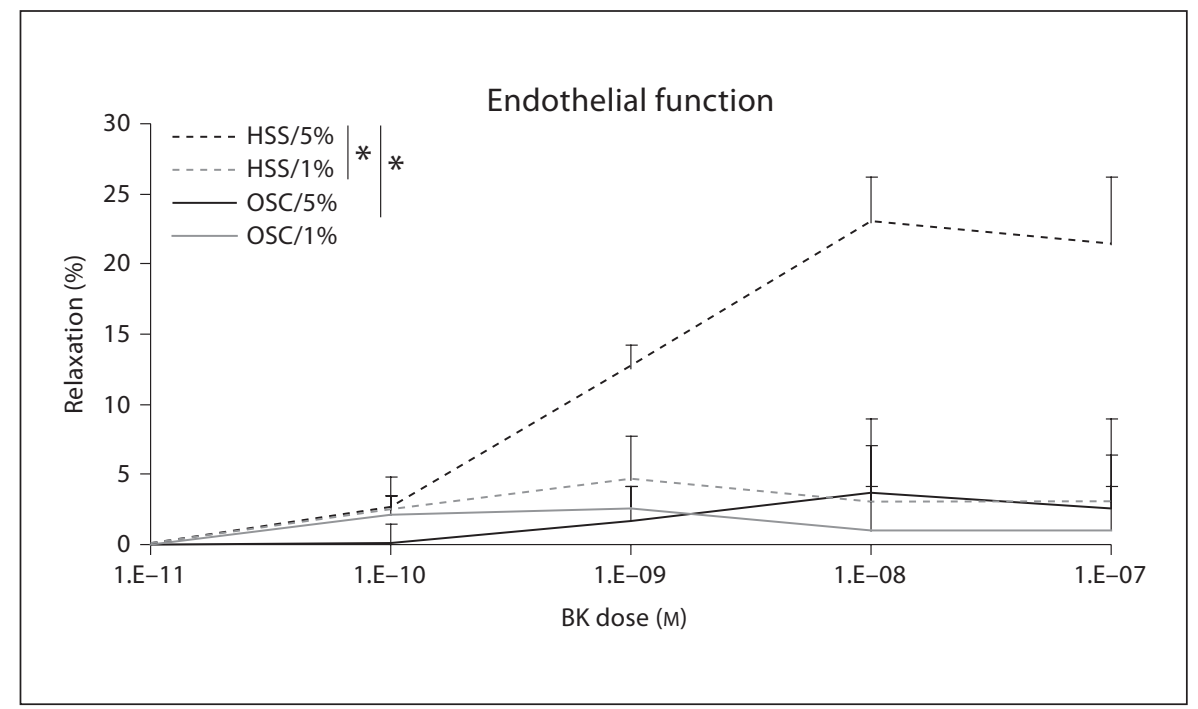

with $5 \mu \mathrm{M}$ DHE (FluoProbes) in PBS, and then rinsed for $1 \mathrm{~min}$ in PBS. Sections were examined on a Leica DM5500 at $20 \times$ magnification. All steps were performed in the dark.

\section{Protein Extraction and Analysis}

Protein expression was assessed using standard Western blot techniques. Protein was extracted from the samples with a Brij35 lyse buffer (50 mM Tris pH 7.5, $1 \mathrm{M} \mathrm{NaCl}, 2 \mathrm{M}$ Urea, 0.1\% Brij35 and 1 protease inhibitor cocktail; Roche). Twenty micrograms of protein were electrophoresed, after which the proteins were transferred to a nitrocellulose filter (Amersham). Filters were incubated with either mouse anti-eNOS (1:1,000; BD Biosciences), mouse anti-nitrotyrosine (1:500; Abcam), mouse anti-eNOS (1:1,000; BD Transduction Laboratories), goat anti-VE-cadherin (1:1,000; Santa Cruz Biotechnology), rabbit anti-p22 ${ }^{\text {phox }}$ (1:200; Santa Cruz Biotechnology), mouse anti-p47phox (1:200; BD Transduction Laboratories), mouse anti-glyceraldehyde-3-phosphate dehydrogenase (GAPDH, 1:1,000; Chemicon International) followed by ECL-peroxidase-labeled anti-mouse, goat or rabbit secondary antibodies (1:5,000; Amersham). Protein expression of eNOS was normalized to the expression of VE-cadherin, while nitrotyrosine, p47-phox and p22-phox were normalized to GAPDH.

\section{Image Analysis}

Images were analyzed using Metamorph (Meta Imaging Series 7.0). For endothelial DHE calculations, we used a WACOM graphic tablet to manually define the endothelial region.

\section{Statistics}

The data are reported as mean values \pm standard deviation. Two-way analysis of variance statistics were performed to assess significant differences between shear stress and cyclic stretch groups. When the interaction among groups was determined to be significant, posttests using the Bonferroni correction were used to assess their interaction. A value of $\mathrm{p}<0.05$ was considered significant.

\section{Results}

Vascular Tissue Reactivity Studies

Endothelial function, as assessed at the $10^{-8}$-M dosage of BK, was significantly modulated by both hemodynamic stimuli shear stress and cyclic stretch. We observed that when changing the flow from HSS to OSC, while maintaining a normal cyclic stretch in both cases, the function of the endothelium decreased by $84 \%$ (fig. 1). When we compared normal and reduced stretch groups where HSS was maintained for both, we observed an $87 \%$ downregulation in endothelial functionality (fig. 1).

\section{Shear Stress, but Not Cyclic Stretch, Modulates eNOS Expression}

Exposure of arteries to OSC significantly decreased the expression of eNOS as compared to HSS. This effect was observed regardless of normal or reduced stretch levels (32 and 28\% decrease, respectively; fig. 2a). Seemingly independent of changes in shear stress, eNOS expression seems to correlate with decreased cyclic stretch; however, results did not reach significance. Results from Western blot analysis were further supported by immunofluorescence of arterial sections. In arteries submitted to HSS, eNOS expression was easily detected on the endothelium, whereas in those submitted to OSC, eNOS expression was barely visible (fig. $2 \mathrm{~b}$ ). 

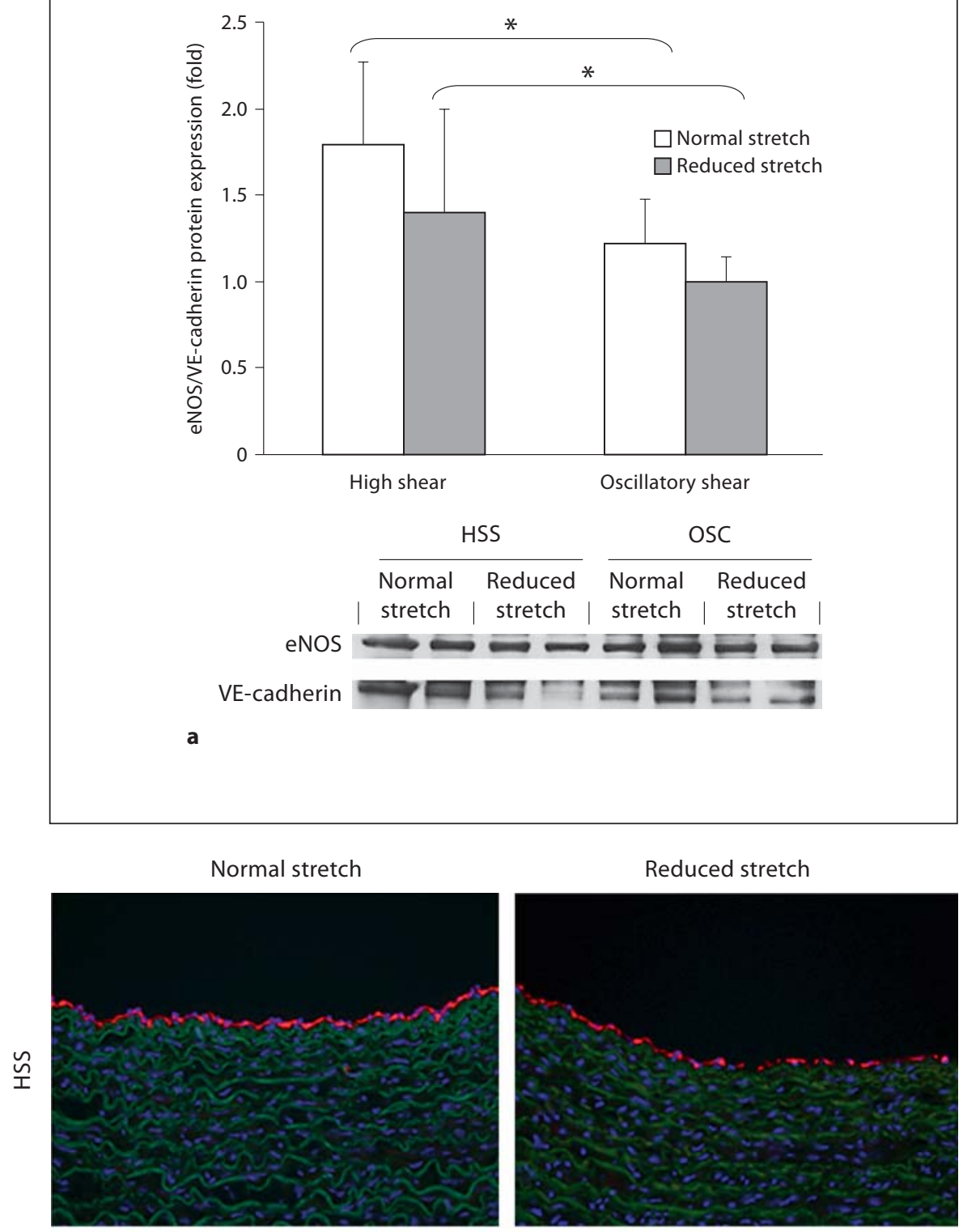

Fig. 2. a The effect of reduced cyclic stretch and oscillatory flow on eNOS expression as evaluated by immunoblot. Data are expressed as means $\pm \mathrm{SD}, \mathrm{n}=6,{ }^{*} \mathrm{p}<0.05$. b eNOS was visualized by immunostaining of arterial cross-sections. Elastin is represented in green, nuclei are represented in blue and eNOS in red. All images were taken at the same contrast and luminescence levels at $20 \times$ magnification.
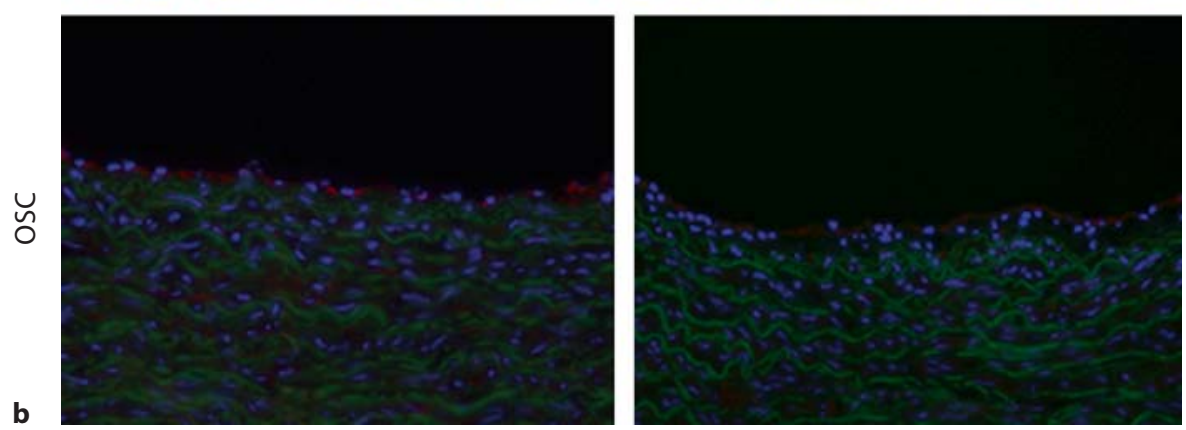

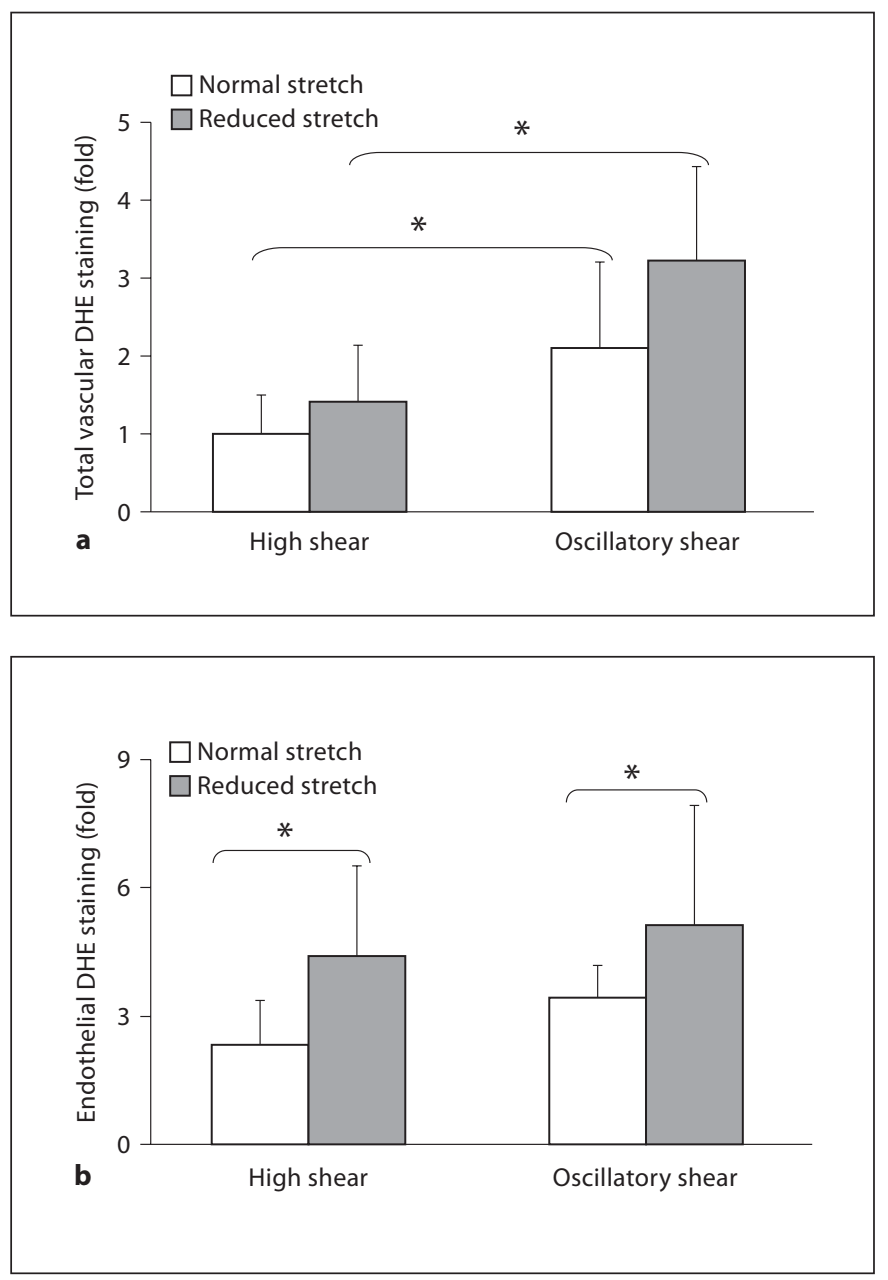

Fig. 3. a The effect of reduced cyclic stretch and oscillatory flow on total ROS expression, as evaluated with DHE staining. Data are expressed as means $\pm \mathrm{SD}, \mathrm{n}=6,{ }^{*} \mathrm{p}<0.001$. b The effect of reduced cyclic stretch and oscillatory flow on endothelial ROS ex-
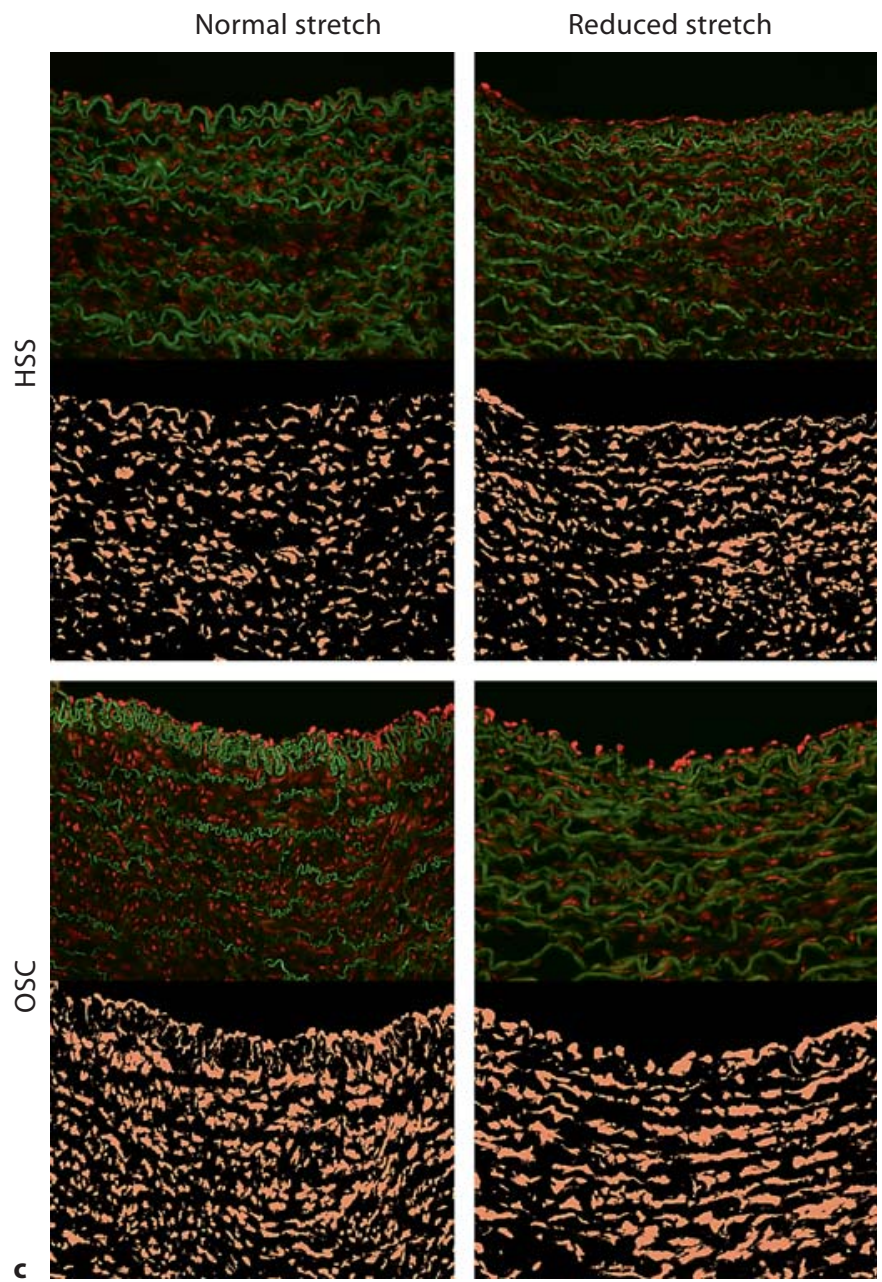

pression, as evaluated with DHE staining. Data are expressed as means $\pm S D, n=6,{ }^{*} p<0.05$. c Sample DHE images showing originals on top (elastin in green, DHE in red) and image after being analyzed with a script written in Metamorph.
Cyclic Stretch and Shear Stress Distinctly Regulate the Production of Superoxide Anion

When looking at total vascular ROS production throughout the endothelial, intimal and medial layers, we observed that ROS is significantly modulated by oscillatory shear stress. Arteries exposed to OSC at both normal and reduced stretch increased 2.1- and 2.3-fold the formation of ROS, respectively, as compared to HSS (fig. 3a). Regarding stretch, we observed a trend indicating that reducing stretch increases ROS independent of shear stress, although these changes were not significant.

Interestingly, when focusing on ROS produced solely by the endothelium, cyclic stretch became the dominant hemodynamic stimuli. Indeed, ROS production is markedly increased from normal to reduced stretch for both HSS and OSC (2- and 1.5-fold increase, respectively; fig. 3b). DHE images representative of those used for analysis have been included (fig. 3c).

\section{Shear Stress Modulates the Expression of p22-phox and 4 47-phox}

Vascular ROS formation is predominantly mediated by the NOX system, which in turn is regulated by the production of p22-phox and p47-phox. Under the same normal stretch condition, OSC increased the expression of $\mathrm{p} 22-$ phox by 1.4-fold as compared to HSS. Increase on p22phox expression by OSC was also seen when arteries were 

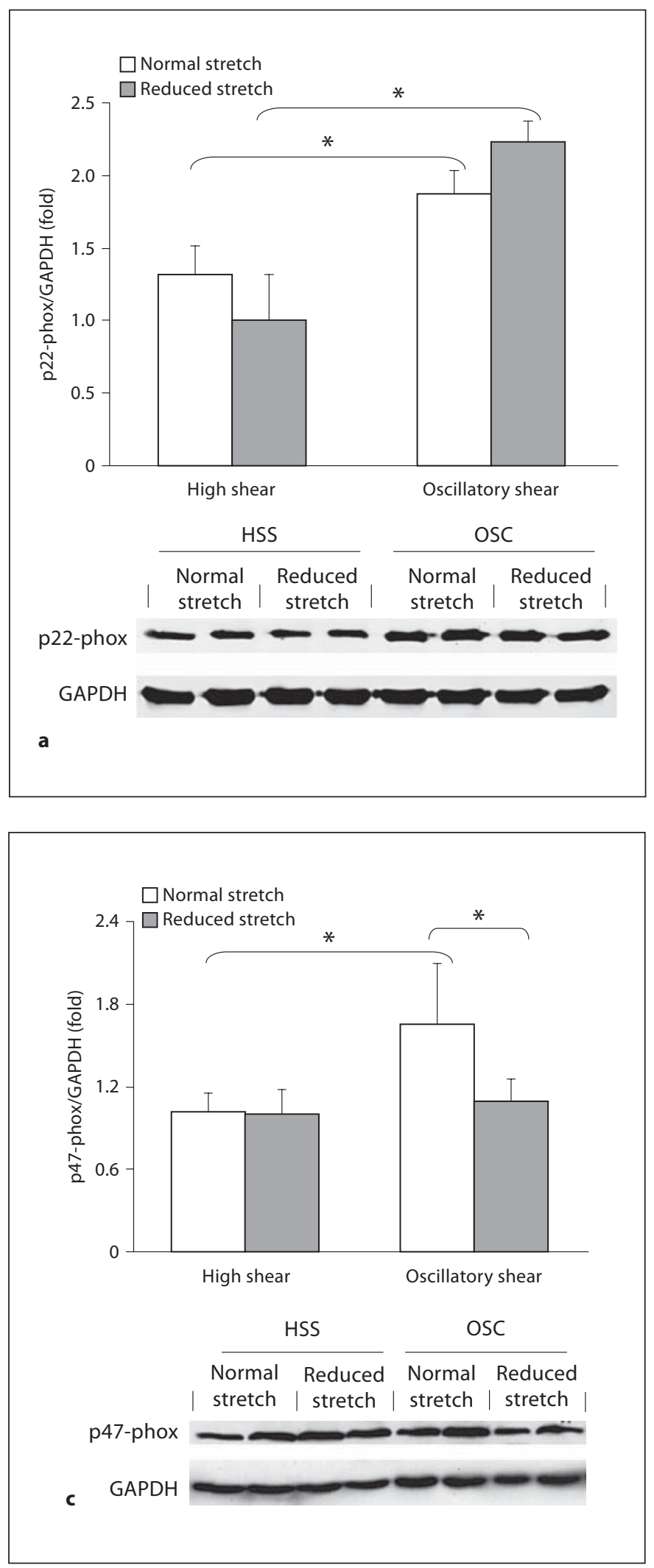

Stretch, Endothelial Dysfunction and Oxidative Stress: An ex vivo Model

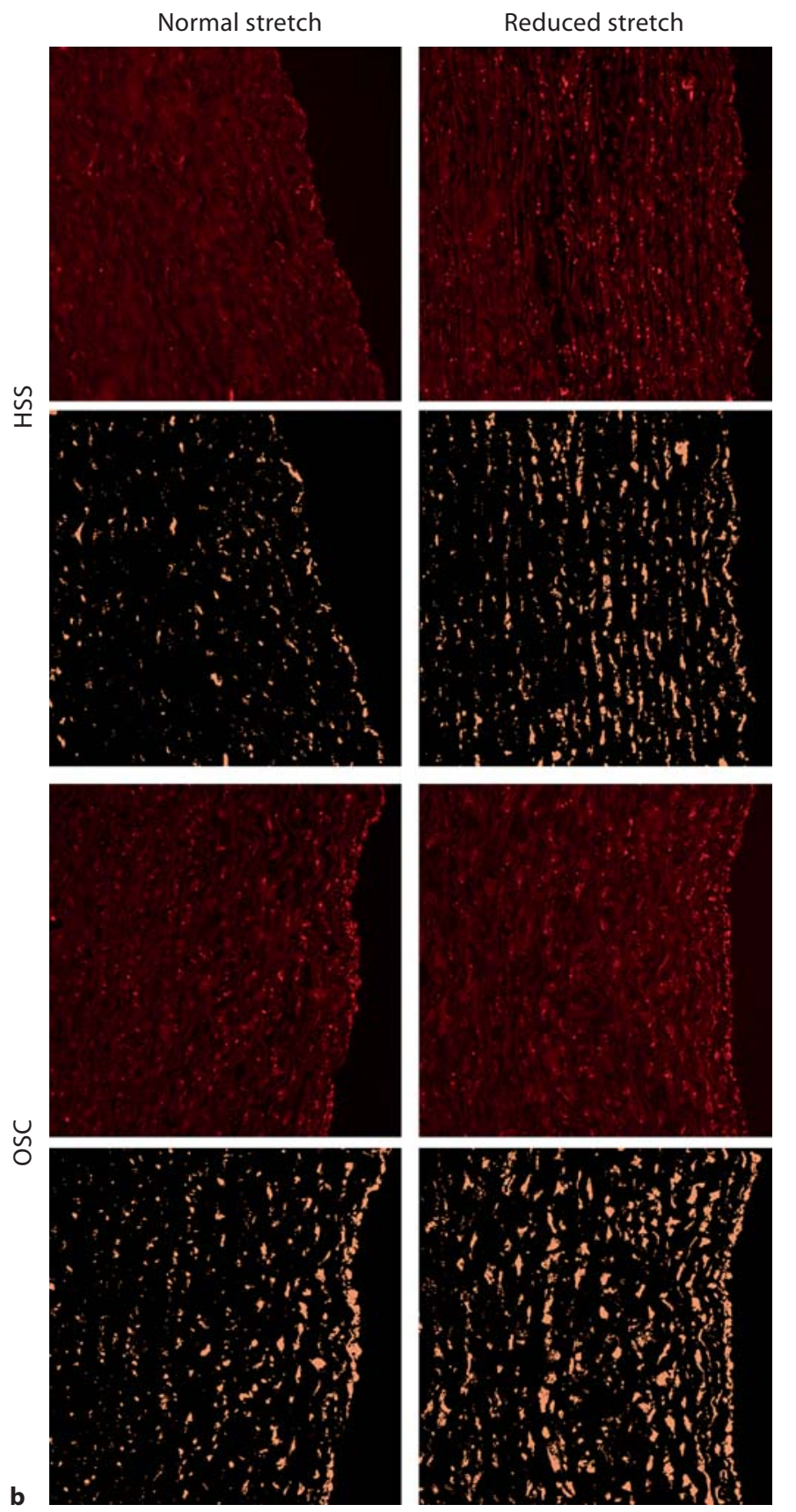

Fig. 4. a The effect of reduced cyclic stretch and oscillatory flow on 22 -phox expression, as evaluated by immunoblot. Data are expressed as means $\pm S D, n=6,{ }^{*} \mathrm{p}<0.05$. b $\mathrm{p} 22$-phox was visualized in red by immunostaining of arterial cross sections. Images on top are originals from just the red channel, while those on the bottom were analyzed with a script written in Metamorph. All images were taken at the same contrast and luminescence levels at $20 \times$ magnification. c The effect of reduced cyclic stretch and oscillatory flow on p47-phox expression, as evaluated by immunoblot. Data are expressed as means $\pm S D, n=6,{ }^{*} \mathrm{p}<0.05$. 
under reduced cyclic stretch condition (2.3-fold upregulation as compared to HSS; fig. 4a). Assessment of p22-phox expression by immunostaining and analysis using Metamorph software allowed us to see the localization of p22phox expression. We observe that reduced stretch tends to increase the expression of p22-phox evenly throughout the arterial wall, whereas OSC predominantly focuses the expression of p22-phox on the endothelium and intimal layers (fig. 4b). The expression of p47-phox was also increased by OSC as compared to HSS. However, in this case the expression was only seen on arteries perfused under normal stretch conditions (fig. 4c).

Shear Stress Mediates the Formation of Nitrotyrosine Increased formation of nitrotyrosine is directly related to the production of peroxynitrite, which is produced when ROS react with NO, thus lowering the vascular bioavailability of NO. Exposure of arteries to OSC under both normal and reduced stretch significantly increased the formation of nitrotyrosine as compared to HSS under the same stretch conditions (1.4- and 1.65-fold upregulation, respectively; fig. 5a). Immunostaining analysis revealed that nitrotyrosine was predominantly located in the intimal and medial layers (fig. 5b).

\section{Discussion}

The current study demonstrates that shear stress and cyclic stretch play significant roles in the progression of endothelial dysfunction and points out their individual and spatial contribution in triggering vascular disease. OSC and reduced stretch both proved detrimental to endothelial function, whereas OSC alone dominated total endogenous vascular wall superoxide anion production. Yet, analysis of oxidative stress in just the endothelial region showed a more pronounced formation of superoxide anion by reduced cyclic stretch than by oscillatory shear. These results emphasize an important distinction between shear- and stretch-mediated effects on the vascular wall. Similarly, Western blot analysis revealed that eNOS and nitrotyrosine expression were also more modulated by oscillatory flow than by reduced stretch. These data suggest that shear and stretch stimulate regions of the vascular wall differently, yet it is their combined effects which contribute to vascular disease.

Previous works have related reduced compliance [16] and oscillatory shear stress [17] to modulation of eNOS expression and endothelial cell homeostasis [18-20]. However, when shear stress and cyclic stretch have been studied in the past, these 2 very important stimuli have often been coupled or studied under such a wide variety of experimental conditions that it has been difficult to determine their individual contributions to vascular disease. In our study we have performed experiments coupling high and oscillatory shear stress with normal and reduced stretch for $72 \mathrm{~h}$, and studied how shear stress and cyclic stretch, 2 very different mechanical forces, can work independently or in concert to evoke atheroprone endothelial responses.

To study if the effects of shear stress and cyclic stretch indeed have an effect on vascular endothelial functionality, we performed vascular reactivity studies on tissue segments from 4 experimental groups: HSS/normal stretch, HSS/reduced stretch, OSC/normal stretch and OSC/reduced stretch. Using the HSS/normal stretch group as reference, we noticed that introducing an OSC

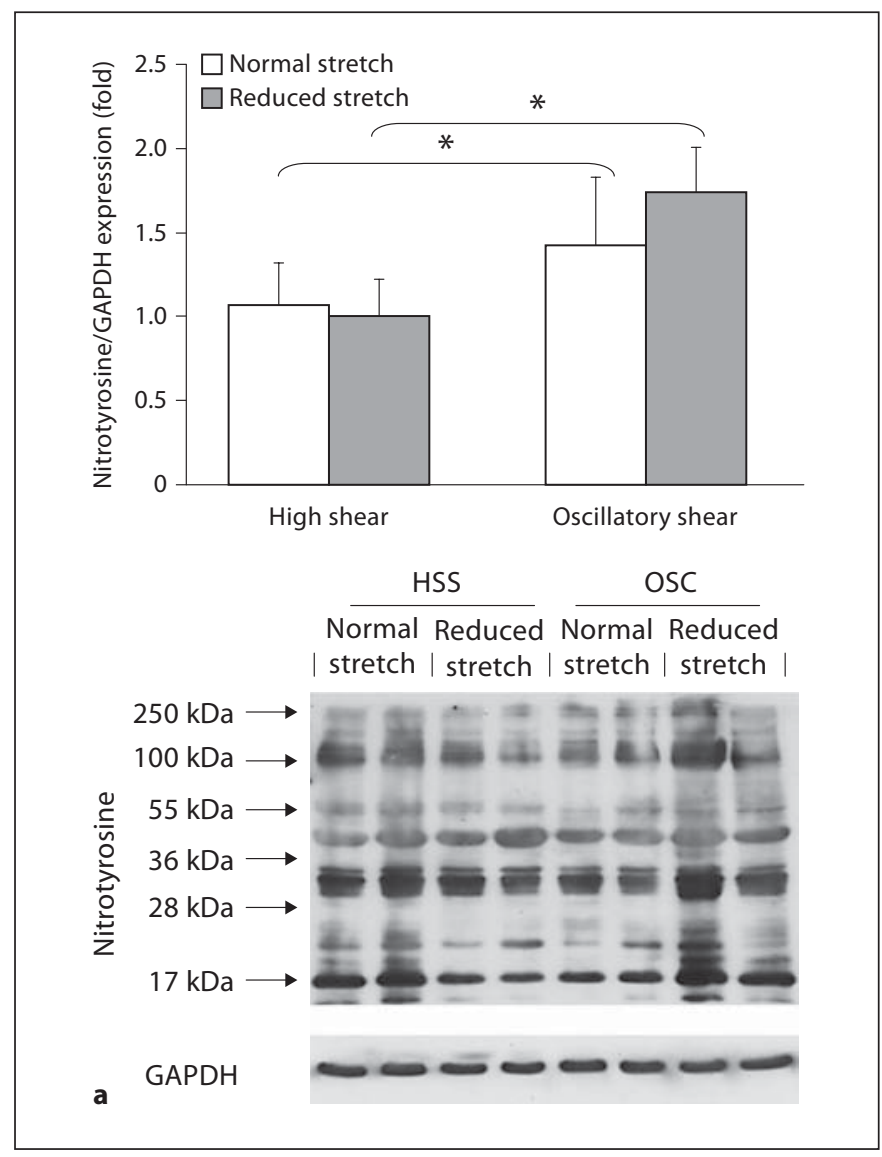

Fig. 5. a The effect of reduced cyclic stretch and oscillatory flow on nitrotyrosine expression, as evaluated by immunoblot. Data are expressed as means $\pm S D, n=6,{ }^{*} \mathrm{p}<0.001$. 
or reducing the stretch both caused decreases in endothelial functionality upon BK stimulation, in the order of $85 \%$. Moreover, the effect of an OSC combined with a reduced stretch nearly completely abolished any sign of endothelial functionality. These results indicate that on a functional level, both shear stress and circumferential stretch are imperative for maintaining endothelial func- tion. Moreover, no significant differences in $\mathrm{KCl}$ contraction or SNP relaxation among the 4 groups were observed, confirming that changes in BK relaxation were due to OSC and reduced stretch and not to loss of vascular smooth muscle function.

To investigate the origins of endothelial dysfunction in regards to shear stress and stretch, we first investigated
Fig. 5. b Nitrotyrosine was visualized in brown by immunostaining on arterial cross-sections using a DAB substrate kit. All images were taken at the same contrast and luminescence levels at $10 \times$ magnification.
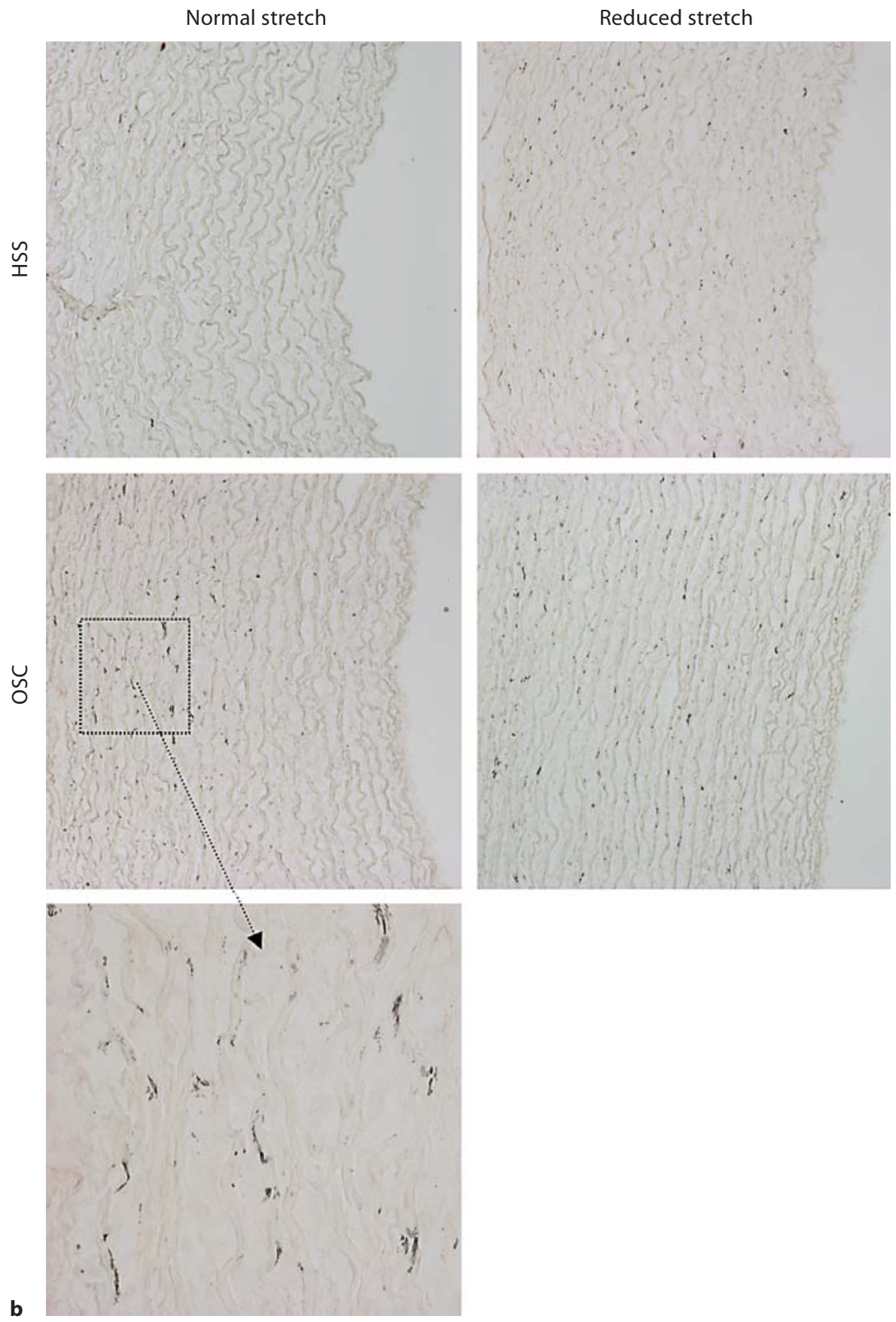

J Vasc Res 2010;47:336-345 
whether eNOS protein expression is modulated differently by reduced cyclic stretch, and oscillatory shear. The most obvious result shows that OSC significantly downregulates eNOS production, which is in agreement with previous findings [21]. Interestingly, there was no statistical difference between the high shear/reduced stretch and the oscillatory shear/normal stretch groups, indicating that in spite of the knowledge that shear stress is a more significant regulator of eNOS, normal stretch conditions are fundamental to retain the protective effects of wall shear stress.

Fluctuations in oxidative stress, namely in the direction of increased levels of ROS, are known to have dramatic effects on endothelial health and vascular function. Basal levels of ROS are essential to vascular cell physiologic function and contribute to the signaling required for vascular remodeling $[22,23]$. However, increases in their production have been shown to decrease vascular levels of NO through reactions forming peroxynitrite [24], causing inflammation and potentially even cell death [25]. Thus, we found it interesting to investigate how shear stress and cyclic stretch modulate ROS production in the vascular wall. To visualize ROS production, arterial cryosections were stained with DHE, a cell-permeable compound which forms ethidium upon reacting with $\mathrm{O}_{2}{ }^{-}$. Quantification was performed by multiplying the percent area for the region of interest of the arterial cross-section expressing ethidium by the average intensity of ethidium expression. It was found that OSC more significantly upregulated total DHE intensity when compared to reduced stretch. Yet, at the endothelial layer, reduced stretch is a more significant modulator of DHE than OSC. These results suggest that reduced stretch and OSC both induce ROS production in the vascular wall, but most importantly, they explain that increases in ROS due to reduced arterial compliance pose a much higher risk to endothelial dysfunction than OSC.

As mentioned above, arterial ROS production is mediated at many levels. The present study focuses on the NOX cascade, known to be a major source of ROS in vascular cells [26]. To measure the activation of the NOX, we investigated the regulation of 2 crucial components, p22-phox and p47-phox.p22-phox was increased by both oscillatory shear and reduced shear stress, but more significantly by oscillatory shear. These results were further supported by immunostaining, which showed that p22-phox expression was highly expressed in the endothelial and intimal region. The expression of the p47-phox component was significantly upregulated in the combination of oscillatory shear and normal stretch. These results suggest that p22-phox and p47-phox could play significant roles in the development of vascular ROS production, but do not necessarily act in concert. At physiologic $\mathrm{pH}$, it is known that high levels of vascular ROS can react with NO to form peroxynitrite, a potent oxidant, thus lowering NO bioavailability. Due to the very short half-life of peroxynitrite, we can measure nitrotyrosine formation, the byproduct of the reaction between peroxynitrite and tyrosine protein residues [9]. The measurement of nitrotyrosine indirectly reflects the amount of $\mathrm{NO}$, which is being removed from the vasculature due to ROS scavenging. Our results showed that oscillatory shear significantly upregulates nitrotyrosine expression, indicating that the increases in ROS due to OSC are causing more reactions with vascular NO, decreasing its bioavailability and creating a more atheroprone endothelial environment.

To conclude, this study demonstrates for the first time in an arterial tissue model the individual contributions of shear stress and cyclic stretch to endothelial injury due to increased superoxide anion production. Our results have shown that OSC is a more dominant stimulus than reduced cyclic stretch with regards to downregulation of eNOS, increased levels of total vascular ROS and nitrotyrosine regulation, the combination of which can be linked to a decreased NO bioavailability. This study also demonstrated the involvement of 2 NOX components, namely p22-phox and p47-phox, in the modulation of ROS expression. Finally, the most interesting finding from this study was that ROS production in the endothelial region is significantly higher and more significantly modulated by reduced stretch than oscillatory shear. This point suggests that although oscillatory shear was shown to more significantly modulate total ROS production, reduced stretch is the more dangerous risk for endothelial injury. These findings agree with results from tissue reactivity studies where we were able to see significant decreases in endothelial functionality due to both OSC and reduced stretch. Because perturbed shear stress and reduced arterial compliance have both been implicated in the initiation and attenuation of arterial disease, this work gives insight to how oscillatory shear stress and reduced compliance provoke endothelial dysfunction and increased risk of atherosclerosis over time.

\section{Acknowledgement}

This work was supported by the Swiss National Science Foundation grant No. 310000118274. 


\section{References}

${ }_{1} \mathrm{Ku}$ DN, Giddens DP, Zarins CK, Glagov S: Pulsatile flow and atherosclerosis in the human carotid bifurcation. Positive correlation between plaque location and low oscillating shear stress. Arteriosclerosis 1985;5:293302.

2 Channon KM, Guzik TJ: Mechanisms of superoxide production in human blood vessels: relationship to endothelial dysfunction, clinical and genetic risk factors. J Physiol Pharmacol 2002;53:515-524.

>3 Glagov S, Zarins C, Giddens DP, Ku DN: Hemodynamics and atherosclerosis. Insights and perspectives gained from studies of human arteries. Arch Pathol Lab Med 1988;112: 1018-1031.

-4 Krams R, Wentzel JJ, Oomen JA, Vinke R, et al: Evaluation of endothelial shear stress and 3D geometry as factors determining the development of atherosclerosis and remodeling in human coronary arteries in vivo. Combining 3D reconstruction from angiography and IVUS (ANGUS) with computational fluid dynamics. Arterioscler Thromb Vasc Biol 1997; 17:2061-2065.

5 Zieman SJ, Melenovsky V, Kass DA: Mechanisms, pathophysiology, and therapy of arterial stiffness. Arterioscler Thromb Vasc Biol 2005;25:932-943.

6 Lehoux S, Tedgui A: Signal transduction of mechanical stresses in the vascular wall. Hy pertension 1998;32:338-345

$\checkmark 7$ Madamanchi NR, Hakim ZS, Runge MS: Oxidative stress in atherogenesis and arterial thrombosis: the disconnect between cellular studies and clinical outcomes. JThromb Haemost 2005;3:254-267.

$\checkmark 8$ Harrison DG, Widder J, Grumbach I, Chen W, et al: Endothelial mechanotransduction, nitric oxide and vascular inflammation. J Intern Med 2006;259:351-363. $\checkmark 9$ MacMillan-Crow LA, Crow JP, Kerby JD, Beckman JS, Thompson JA: Nitration and inactivation of manganese superoxide dismutase in chronic rejection of human renal allografts. Proc Natl Acad Sci USA 1996;93: 11853-11858.

10 Michell BJ, Harris MB, Chen ZP, Ju H, et al: Identification of regulatory sites of phosphorylation of the bovine endothelial nitricoxide synthase at serine 617 and serine 635 . J Biol Chem 2002;277:42344-42351.

11 Bae SW, Kim HS, Cha YN, Park YS, et al: Rapid increase in endothelial nitric oxide production by bradykinin is mediated by protein kinase A signaling pathway. Biochem Biophys Res Commun 2003;306:981987.

12 Valko M, Leibfritz D, Moncol J, Cronin MT, et al: Free radicals and antioxidants in normal physiological functions and human disease. Int J Biochem Cell Biol 2007;39:44-84.

$>13$ Taubman MB, Fallon JT, Schecter AD, Giesen $\mathrm{P}$, et al: Tissue factor in the pathogenesis of atherosclerosis. Thromb Haemost 1997; 78:200-204.

14 Montorzi G, Silacci P, Zulliger M, Stergiopulos N: Functional, mechanical and geometrical adaptation of the arterial wall of a nonaxisymmetric artery in vitro. J Hypertens 2004;22:339-347.

15 Gambillara V, Thacher T, Silacci P, Stergiopulos N: Effects of reduced cyclic stretch on vascular smooth muscle cell function of pig carotids perfused ex vivo. Am J Hypertens 2008;21:425-431.

16 Qiu Y, Tarbell JM: Interaction between wall shear stress and circumferential strain affects endothelial cell biochemical production. J Vasc Res 2000;37:147-157.

-17 Noris M, Morigi M, Donadelli R, Aiello S, et al: Nitric oxide synthesis by cultured endothelial cells is modulated by flow conditions. Circ Res 1995;76:536-543.

18 Peng X, Haldar S, Deshpande S, Irani K, Kass DA: Wall stiffness suppresses Akt/eNOS and cytoprotection in pulse-perfused endothelium. Hypertension 2003;41:378-381.
19 Li Y, Zheng J, Bird IM, Magness RR: Effects of pulsatile shear stress on signaling mechanisms controlling nitric oxide production, endothelial nitric oxide synthase phosphorylation, and expression in ovine fetoplacental artery endothelial cells. Endothelium 2005;12:21-39.

20 Ziegler T, Silacci P, Harrison VJ, Hayoz D: Nitric oxide synthase expression in endothelial cells exposed to mechanical forces. Hypertension 1998;32:351-355.

-21 Gambillara V, Montorzi G, Haziza-Pigeon C, Stergiopulos N, Silacci, P: Arterial wall response to ex vivo exposure to oscillatory shear stress. J Vasc Res 2005;42:535-544.

22 Skyschally A, Schulz R, Gres P, Korth HG, Heusch G: Attenuation of ischemic preconditioning in pigs by scavenging of free oxyradicals with ascorbic acid. Am J Physiol Heart Circ Physiol 2003;284:H698-H703.

23 Chen W, Gabel S, Steenbergen C, Murphy E: A redox-based mechanism for cardioprotection induced by ischemic preconditioning in perfused rat heart. Circ Res 1995;77:424429 .

24 Baldus S, Eiserich JP, Brennan ML, Jackson RM, et al: Spatial mapping of pulmonary and vascular nitrotyrosine reveals the pivotal role of myeloperoxidase as a catalyst for tyrosine nitration in inflammatory diseases. Free Radic Biol Med 2002;33:1010.

25 Taylor BM, Fleming WE, Benjamin CW, Wu $\mathrm{Y}$, et al: The mechanism of cytoprotective action of lazaroids I: inhibition of reactive oxygen species formation and lethal cell injury during periods of energy depletion. J Pharmacol Exp Ther 1996;276:1224-1231.

26 Griendling KK, Sorescu D, Ushio-Fukai M: NAD(P)H oxidase: role in cardiovascular biology and disease. Circ Res 2000;86:494501 . 